\title{
Trauma - Unterschätzte Verbreitung und Langzeitfolgen
}

\author{
Nadine Wickert • David Mayrhofer
}

Angenommen: 10. September 2021 / Online publiziert: 1. Oktober 2021

(C) Springer Fachmedien Wiesbaden GmbH, ein Teil von Springer Nature 2021

Der Begriff Trauma spielt in den Disziplinen, die sich mit psychischer Gesundheit und Krankheit beschäftigen, seit jeher eine wichtige Rolle und wurde - je nach Schule und deren Menschenbild und Verständnis von Pathogenese - auf vielfältige Weise definiert. Anders als bei anderen psychischen Störungen haben sich rund um das Phänomen Trauma eigenständige Methoden und therapeutische Ansätze entwickelt, die unter dem Begriff der Psychotraumatologie zusammengefasst werden und sehr oft einen interdisziplinären Ansatz verfolgen. Weithin bekannt sind etwa die Psychodynamisch Imaginative Traumatherapie (PITT) von Luise Reddemann (2004), die Ego-State-Therapie von John und Helen Watkins (1997) und das Eye Movement Desensitization and Reprocessing (EMDR) von Francine Shapiro (1995), um hier nur einige zu nennen.

Das Wissen und Verständnis über Traumata wird heute über die Grenzen der Disziplinen hinweg geteilt. Ein wesentlicher Baustein dieses Wissens ist, dass Traumata nicht durch das Erfahren von schlimmen Ereignissen selbst entstehen, sondern in der psychischen Verarbeitung dieser Erfahrungen begründet sind. Als man entdeckte, dass die gleichen Ereignisse (Katastrophen, Kriegserfahrungen, Missbrauchserfahrungen etc.) nicht bei allen Menschen, die diese erlebten, zu Traumafolgestörungen führen, hat das auch das Interesse an der Erforschung von Resilienz beflügelt (vgl. dazu auch das ZPS-Heft Resilienz von Wickert und Meents 2020). In der Praxis schlägt sich das in der starken Gewichtung der Ressourcenorientierung in allen

\footnotetext{
Nadine Wickert $(\bowtie)$

Praxis Ungargasse, Ungargasse 21/1, 1030 Wien, Österreich

E-Mail: mail@psychotherapie-wickert.at

David Mayrhofer

Währinger Straße 132/10, 1180 Wien, Österreich

E-Mail: praxis@david-mayrhofer.at
} 
modernen traumatherapeutischen Ansätzen nieder. Gerade in diesem Punkt hat das Psychodrama - als eine entwicklungsorientierte Methode - einiges beizutragen.

Die große Bandbreite an Traumafolgestörungen und damit verbundenen Gesundheitsbeeinträchtigungen stellen - man muss es so drastisch sagen - eines der größten, weitreichendsten und kostspieligsten Gesundheitsprobleme unserer Gesellschaft dar, auch wenn dies gemeinhin eher übersehen wird. Eine evidenzbasierte Untermauerung dieser Behauptung stellen die Ergebnisse der bahnbrechenden Untersuchung über die Auswirkungen von schädlichen Kindheitserfahrungen des US-amerikanischen Präventivmediziners Vincent Felitti und des US-amerikanischen Epidemiologen Robert Anda dar, die sogenannte ACE-Studie (ACE= Adverse Childhood Experiences, vgl. Felitti et al. 1998).

Felitti arbeitete in den 1980er-Jahren in einer Adipositas-Klinik in San Diego. Dort stellte er bald fest, dass auffällig viele seiner stark übergewichtigen Patientinnen und Patienten eine Gemeinsamkeit hatten: Sie waren in ihrer Kindheit sexuell missbraucht oder mit anderen schwerwiegenden Problemen konfrontiert worden. Das Thema ließ ihn nicht mehr los, und gemeinsam mit dem Epidemiologen Robert Anda führte er eine breit angelegte Studie durch. So wollten sie den Zusammenhang zwischen belastenden Erfahrungen in der Kindheit und Erkrankungen im Erwachsenenalter sowie gesundheitsgefährdendes Risikoverhalten von Erwachsenen erforschen (vgl. van der Kolk 2021, S. 175 f.).

Über 17.000 erwachsene Patientinnen und Patienten befragten sie schließlich zu belastenden Erfahrungen in deren Kindheit und Jugend (z. B. körperlicher, psychischer oder sexueller Missbrauch bzw. Vernachlässigung, Kontakt mit häuslicher Gewalt, Suchtmittelmissbrauch oder psychische Erkrankungen im Haushalt, Trennung oder Scheidung der Eltern, inhaftiertes Haushaltsmitglied) und glichen die Antworten mit detaillierten medizinischen Langzeitdaten und Befunden derselben PatientInnen ab. Die Ergebnisse, die sie 1998 erstmals veröffentlichten, waren erschreckend: Nicht nur fanden sie heraus, dass traumatische Kindheitserfahrungen weit verbreitet sind, sondern auch, dass diese mitunter beträchtliche lebenslange Folgewirkungen verursachen und einen starken Einfluss auf die spätere geistige wie auch körperliche Gesundheit der betroffenen Personen haben (Felitti et al. 1998).

Mehr als 50\% der damals Befragten gaben an, mindestens eine belastende Kindheitserfahrung bzw. ein ACE (Adverse Childhood Experience) erlebt zu haben. Bei mehr als 6\% der Befragten waren es sogar vier oder mehr ACEs. Mit ihrer Studie konnten Felitti und Anda auch aufzeigen, dass Menschen, die mehrere ACEs erlebt hatten, im Vergleich zu Menschen ohne belastende Kindheitserfahrung ein deutlich gesteigertes Risiko für eine Vielzahl von gesundheitlichen und psychischen Folgeerkrankungen haben. Beispielhaft seien hier nur einige genannt: Menschen mit vier oder mehr ACEs haben im Vergleich zu Menschen ohne ACE ein vier- bis 12-fach erhöhtes Risiko für Alkoholismus, Drogenmissbrauch, Depressionen oder Suizidversuche. Sie haben ein doppelt so hohes Risiko für koronare Herzerkrankungen, ein 1,9-fach erhöhtes Risiko für Krebserkrankungen, ein 3,9-fach erhöhtes Risiko für chronische Bronchitis etcpp. (Felitti et al. 1998). In einer Folgeuntersuchung aus dem Jahr 2009 wurde gar festgestellt, dass Menschen mit sechs oder mehr ACEs eine insgesamt fast 20 Jahre geringere Lebenserwartung haben als Menschen ohne ACE (Brown et al. 2009). 
In einer Studie von 2019 - also mehr als 20 Jahre nach Erscheinen der ACEStudie - wurde nachgewiesen, dass die Ergebnisse auch in der gegenwärtigen deutschen Bevölkerung nahezu ident ausfallen (Witt et al. 2019). Die WHO geht davon aus, dass ähnliche Ergebnisse wie die der ACE-Studie auch in Ländern mit unterschiedlichem wirtschaftlichem und sozialem Entwicklungsstand zu finden sind, sprich, dass die Lage also weltweit eine ähnliche ist (WHO 2006). 2019 erschien zudem eine Meta-Studie der WHO, die 23 Artikel systematisch reviewte und daraus die jährlichen Gesamtkosten der Sozial- und Gesundheitssysteme in Europa und Nordamerika errechnete, die eindeutig ACEs zuzuschreiben sind. Für Europa wurden jährliche Kosten in Höhe von etwa 490 Mrd. $€$ geschätzt. Mehr als $75 \%$ dieser Kosten entstanden bei Personen mit zwei oder mehr ACEs (Bellis et al. 2019).

Dank der Fortschritte der Neurowissenschaften weiß man auch um die Hintergründe dieser drastischen Folgewirkungen auf die Gesundheit (Weiss und Wagner 1998; Danese und McEwen 2012). Sind Kinder belastenden Ereignissen ausgesetzt und werden diese nicht ausreichend dabei unterstützt, ihre Stressreaktionen zu regulieren, so beeinflusst die Überlastung des Nervensystems mit Stresshormonen nachhaltig die Entwicklung des Gehirns. Geringere Stressresistenz und häufigere Stressreaktionen sind die nachhaltige Folge.

Hier sei angemerkt, dass - wie eingangs schon erwähnt - belastende Erfahrungen nicht zwangsweise bei jedem Menschen zu einer Traumafolgestörung führen. Im Einzelfall kann man ein ACE nicht mit einem Trauma gleichsetzen. Dennoch muss man ACEs als potenziell traumatisierend verstehen. Kinder sind bei Belastungen auf die Koregulation durch Bezugspersonen angewiesen und damit viel schneller Hilflosigkeitsempfinden, das mit einer Traumatisierung einhergeht, ausgesetzt.

Obgleich all diese Untersuchungen in Summe sehr beunruhigend sind und Anlass für eine breit angelegte Diskussion und für Aufklärungs- und Präventionsmaßnahmen geben sollten, sind die Erkenntnisse - zumindest ist dies unsere Wahrnehmung als HerausgeberInnen dieses Sonderbands - weder in der allgemeinen Bevölkerung noch in Fachkreisen (PsychotherapeutInnen, MedizinerInnen, PsychologInnen, aber auch PolitikerInnen, LehrerInnen etcpp.) sonderlich bekannt. Dies ist besonders erstaunlich, liegen doch die Evidenzen schon seit Jahrzehnten vor.

Grund genug also, in der Zeitschrift für Psychodrama und Soziometrie einen Sonderband zum Thema Trauma zu veröffentlichen. Schon 2002 war ein reguläres ZPSHeft mit dem Titel Trauma, Störung und Ressource erschienen (vgl. Krüger und Weber 2002). Seither haben sich Traumatologie und auch die psychodramatische Traumatheorie und -therapie stetig weiter entwickelt. Mit dem vorliegenden Sonderband wollen wir einen breiten Überblick über die aktuelle Forschungslage, über psychodramatische und auch über einige Behandlungszugänge anderer Verfahren geben. Dafür kommen neben einer Vielzahl von Psychodramatikerinnen und Psychodramatikern auch mehrere namhafte Wegbereiterinnen und Wegbereiter der Psychotraumatologie zu Wort. Aufgrund der Interdisziplinarität der Psychotraumatologie haben wir uns bei der Auswahl der Beiträge für diesen Sonderband interdisziplinärer orientiert als es für die ZPS üblich ist.

Sonja Hintermeier gibt uns einleitend einen Überblick über die Klassifikation von Trauma und Traumfolgestörungen und widmet sich dann der Frage, wie kindliche Traumatisierung und strukturelle Störungen zusammenhängen. Sie beschreibt, 
wie sich Traumafolgestörungen im Kindesalter zu strukturellen Störung entwickeln können und erklärt, welche strukturellen Störungen auf Traumatisierungen zurückzuführen sind.

Darauf folgt ein Beitrag von Hildegard Pruckner, in dem sie uns einerseits einen kompakten Überblick über die Arbeitsweise des humanistisch orientierten Psychodramas gibt und andererseits eine sehr praxisorientierte Beschreibung von störungsspezifischen Modifikationen in der Arbeit mit traumatisierten Menschen bietet. Anhand von anschaulich beschriebenen Fallbeispielen werden wesentliche Fragen der praktischen Arbeit inklusive der speziellen Rollengestaltung beantwortet.

Kurt Fellöcker geht in seinem Artikel der Frage nach, wie die Kombination von Traumafolgestörung und Suchterkrankung psychotherapeutisch behandelt werden kann. Mit Verweis auf empirische Studien erarbeitet er eine State-of-the-art-Perspektive und plädiert dabei für eine integrative Behandlung beider Störungen, was bedeutet, dass der Fokus der Therapie auf Sucht und Trauma gleichermaßen gerichtet sein soll. Er erörtert unter anderem anhand eines Fallbeispiels die Bedeutung eines stabilisierenden, ressourcenorientierten utilisierenden Ansatzes.

Im folgenden Beitrag widmet sich Katharina Chalupsky dem Thema der Trauer und der Trauerarbeit in der Psychotherapie. Beide Themen sind mitunter eng miteinander verknüpft, weshalb es wichtig und sinnvoll ist, für die Arbeit an Traumata auch Kenntnisse über Trauerarbeit zu haben, als auch umgekehrt über Traumakenntnisse in der Arbeit mit Trauernden zu verfügen.

Axel Eichel gibt uns einen Einblick in die Arbeitsweise mit dem Therapeutischen Spiralen Modell, einem traumaspezifischen, psychodramatischen Behandlungsansatz, der speziell für die Gruppenarbeit entwickelt wurde. Anhand eines Fallbeispiels werden die traumaspezifischen Strukturen, Interventionen und Sichtweisen dieses Modells veranschaulicht.

Angelika Eisterer und Sabine Dungl-Nemetz haben sich in ihrem Artikel an ein Verfahren übergreifendes Projekt gewagt und beschreiben sowohl aus psychodramatischer, als auch aus existenzanalytischer Perspektive das Konzept und die angewendeten Interventionen eines ambulanten gruppentherapeutischen Angebots für (komplex) traumatisierte Menschen. Damit die Arbeit im ambulanten Gruppensetting mit dieser Klientel möglich und sinnvoll gestaltet werden kann, wird die Bedeutung von wichtigen Elementen veranschaulicht (z. B. angemessene Rahmenbedingungen, Grundhaltungen, Gruppenkohäsion etc).

Darauf folgt ein praktisch orientierter Artikel von Susanne Neureiter-Penn. Sie stellt die Intervention der Ressourcen-Insel vor, die sich für die Arbeit mit Menschen mit Traumafolgestörungen besonders eignet. Bei dieser Intervention gestalten TeilnehmerInnen im Gruppensetting ihre persönliche Ressourcen-Insel und bringen dabei die eigenen Ressourcen mittels einer Aufstellung von Intermediärobjekten auf die Bühne. Adaptierungsmöglichkeiten für das Einzelsetting werden aufgezeigt.

Doris Pinz-Cronenberg stellt anhand von Fallbeispielen dar, wie psychodramatische Interventionen im Jugendstrafvollzug mit Gewalttätern eingesetzt werden können. Vor allem der Einsatz der Tischbühne und der Lebenslinie werden beschrieben und als eine Möglichkeit aufgezeigt, eine (tiefenpsychologische) Therapie zu unterstützen. 
Martina McClymont-Nielitz und Reinhard Krüger berichten in ihrem Artikel von ihren Erfahrungen mit einem Online-Seminar für TherapeutInnen und BeraterInnen, in dem es um die Vermittlung störungsspezifischer Psychodramatherapie bei Traumfolgestörungen geht. Es werden Psychodramatechniken vorgestellt, die online eingesetzt werden können und darauf abzielen, die metakognitive Störung der Konfliktverarbeitung bei Trauma-PatientInnen aufzulösen.

Silke Gahleitner, Adrian Golatka, Christina Rothdeutsch-Granzer und Helmut Kronberger geben uns in ihrem Artikel einen Einblick in die Entstehung und Grundpfeiler der Traumapädagogik, die im Vergleich zu Traumaberatung und -therapie noch weniger etabliert ist. Der Beitrag gibt einen Einblick in das Drei-PhasenModell der Traumapädagogik und zeigt das Potenzial eines Dialogs zwischen Psychodrama und Traumapädagogik auf.

Mit der frühesten Form von Traumatsierung beschäftigt sich Gabriele BieglerVitek. Sie beleuchtet in ihrem Artikel Formen der Traumatisierung, die während der intra-uterinen Zeit und während der Geburt entstehen können. Mit Blick auf die Ergebnisse der Pränatalforschung beschreibt die Autorin welche Interventionsrichtlinien sich für die psychodramatische Behandlung von Säuglingen, Kindern, Jugendlichen und Erwachsenen ableiten lassen. Anhand von zwei Fallbeispielen verdeutlicht sie, wie die Traumata als im Körpergedächtnis gespeichert verstanden werden und die späteren Lebensszenen der Betroffenen (mit-)bestimmen können.

Anschaulich zeigt Ulrike Altendorfer-Kling in ihrem Artikel, wie digitale Medien in der Psychodramatherapie verwendet werden können. Anhand von Fallvignetten beschreibt sie, wie Smartphone und Co. zum Beispiel für die Umsetzung von klassisch-psychodramatischen Techniken wie Doppeln, Spiegeln, Rollenwechsel u.a. eingesetzt werden können.

Dorothea Weinberg stellt in einem gemeinsamen Artikel mit Nadine Wickert das Konzept und die Entwicklung der Traumabezogenen Spieltherapie (TbSpTh) vor, wobei die Zweitautorin den Konnex zur psychodramatischen Theorie herstellt. Mit dem Verfahren können früh- und komplextraumatisierte Kinder behandelt werden. Beispielhaft werden die Interventionen Strukturierte Traumaintervention (STI), Wunscherfüllende Spiele bzw. das Traumspiel beschrieben.

Maria Helbich widmet sich in ihrem Artikel einer kritischen Betrachtung der Phänomene Dekontextualisierung und Depolitisierung in der psychotherapeutischen Disziplin. Nach einer Beschreibung der Lebenssituation in Palästina und den besetzten Gebieten greift die Autorin das Thema der kollektiven Traumatisierung auf und kommt so zur Frage der gesellschaftspolitischen Bedeutung von Psychotherapie. Sie beschreibt, welches Potenzial Psychodrama in einem Kontext hat, in dem die vorherrschende westliche Haltung Traumatisierungen als Einzelschicksal sieht.

Über den Tellerrand blicken wir mit dem Artikel von Albert Lichtblau, der uns als Historiker einen Einblick in die Methode Oral History gibt, die sich mit der Aufzeichnung, Archivierung und Auswertung von lebensgeschichtlichen Interviews befasst. Nach einer Einführung in die Methode, reflektiert er in anschaulicher und sehr persönlicher Form die Berührungspunkte, an denen Psychotherapie und Oral History in einen fruchtbaren Dialog treten können.

Florian Dunkel nähert sich dem Thema der transgenerationalen Traumatisierung aus dem psychotherapeutischen Blickwinkel und gibt einen anschaulichen Überblick 
über den Stand der Forschung, inklusive ihrer Lücken. Er geht auf die Frage ein, wie das Phänomen in seiner Entstehung verstanden werden kann und welche Behandlungsansätze es gibt.

Christine Pichlhöfer erörtert in ihrem Artikel, welche Kompetenzen TherapeutInnen brauchen, um gut mit Menschen mit Fluchterfahrungen arbeiten zu können. Sie thematisiert Fragen von interkultureller Kompetenz, bei der es um Wissensaneignung, Reflexionsvermögen und Sensibilität geht, um nicht voreingenommen zu agieren und auf Augenhöhe einen stützenden therapeutischen Prozess initiieren zu können.

Romana Tripolt stellt uns in ihrem Artikel die von ihr entwickelte IBT (Integrative Bewegte Traumatherapie) und das Gruppenformat Tanz aus dem Trauma vor. In diesem Ansatz wird Bewegung im Rahmen der Therapie als Ressource, Medium und Anker genutzt. Die Autorin ist Psychodramatikerin und beschreibt wie psychodramatische Elemente in das von ihr entwickelte Gruppenformat Eingang gefunden haben.

Einen fundierten Überblick über körperorientierte und bifokale Behandlungsansätze gibt Oliver Schubbe. Er stellt folgende Methoden vor: Somatic Experiencing (SE), Eye Movement Desensitization and Reprocessing (EMDR), Brainspotting (BSP), Progressive Counting (PC) und Imagery Rescripting (ImRs, IRRT).

Luise Reddemann gibt uns in ihrem Artikel einen Einblick darüber, wie sie aus der von ihr entwickelten Psychodynamisch Imaginativen Traumatherapie heraus den Begriff des Täterintrojektes versteht und wie sie selbst in der Therapie mit diesem Phänomen arbeitet. Die theoretische Basis der Begriffe Ego States und Introjekte wurde von den Psychoanalytikern Paul Federn und Sándor Ferenczi gelegt, was von der Autorin erörtert und mit der Entwicklung ihres Behandlungsansatzes in Beziehung gesetzt wird.

Die Trauma-Expertin Michaela Huber zeigt in ihrem Beitrag Wege zur Behandlung von Menschen mit einer Dissoziativen Identitätsstörung (DIS) auf. Die meisten Menschen mit einer Dissoziativen Identitätsstörung haben langjährige und tiefgreifende Erfahrungen von Gewalt, Vernachlässigung und Missbrauch seit frühester Kindheit gemacht. Die Autorin empfiehlt in ihrem Artikel ein bindungsorientiertes Vorgehen in der Psychotherapie von Menschen mit DIS und stellt konkret zehn Behandlungsaspekte vor, die zur Symptomverbesserung und Stärkung der Persönlichkeitsstruktur beitragen können.

Wir hoffen, mit dieser Fülle an AutorInnen und Themen das Interesse unserer LeserInnenschaft geweckt zu haben und somit einen kleinen Beitrag zur Verbreitung von Wissen zu diesem äußert relevanten Thema zu leisten. Im Namen der gesamten ZPS-Redaktion wünschen wir Ihnen eine anregende Lektüre!

Nadine Wickert und David Mayrhofer

\section{Literatur}

Bellis, M. A., Hughes, K., Ford, K., Ramos Rodriguez, G., Sethi, D., \& Passmore, J. (2019). Life course health consequences and associated annual costs of adverse childhood experiences across Europe and North America: a systematic review and meta-analysis. Lancet Public Health, 4(10), e517-e528. https://doi.org/10.1016/S2468-2667(19)30145-8. 
Brown, D. W., Anda, R.F., Tiemeier, H., Felitti, V. J., Edwards, V. J., Croft, J. B., \& Giles, W. H. (2009). Adverse childhood experiences and the risk of premature mortality. Am J Prev Med, 37(5), 389-396. https://doi.org/10.1016/j.amepre.2009.06.021.

Danese, A., \& McEwen, B.S. (2012). Adverse childhood experiences, allostasis, allostatic load, and agerelated disease. Physiology \& Behavior, 106(1), 29-39. https://doi.org/10.1016/j.physbeh.2011.08. 019.

Felitti, V.J., Anda, R.F., Nordernberg, D., Willimason, D.F., Spitz, A. M., Edwards, V., Koss, M. P., \& Marks, J.S. (1998). Relationship of childhood abuse to many of the leading causes of death in adults. The adverse childhood experiences (ACE) study. American Journal of Preventative Medicine, 14, 245-258. https://doi.org/10.1016/S0749-3797(98)00017-8.

van der Kolk, B. (2021). Verkörperter Schrecken. Traumaspuren in Gehirn, Geist und Körper und wie man sie heilen kann. Paderborn: Probst.

Krüger, R., \& Weber, K. (Hrsg.). (2002). Trauma, Störung und Ressource. Zeitschrift für Psychodrama und Soziometrie, Bd. 2/2002. Wiesbaden: Springer VS.

Reddemann, L. (2004). Psychodynamisch Imaginative Traumatherapie: PITT ${ }^{\circledR}$ - Das Manual. Stuttgart: Klett-Cotta.

Shapiro, F. (1995). Eye movement desensitization and reprocessing: basic principles, protocols, and procedures. New York: Guilford.

Watkins, J.G., \& Watkins, H.H. (1997). Ego states. Theory and therapy. New York: W. W. Norton.

Weiss, M.J., \& Wagner, S.H. (1998). What explains the negative consequences of adverse childhood experiences on adult health? Insights from cognitive and neuroscience research. American Journal of Preventive Medicine, 14(4), 356-360. https://doi.org/10.1016/S0749-3797(98)00011-7.

Wickert, N., \& Meents, A. (Hrsg.). (2020). Resilienz. Zeitschrift für Psychodrama und Soziometrie, Bd. $1 / 2020$.

Witt, A., Sachser, C., Plener, P.L., Brähler, E., \& Fegert, J.M. (2019). The prevalence and consequences of adverse childhood experiences in the German population. Dtsch Arztebl Int, 116, 635-642. https:// doi.org/10.3238/arztebl.2019.0635.

World Health Organization (2006). Preventing child maltreatment: a guide to taking action and generating evidence / World Health Organization and International Society for Prevention of Child Abuse and Neglect. https://apps.who.int/iris/handle/10665/43499. Zugegriffen am 5. Juli 2021. 